\title{
Entwicklung der Notaufnahmefallzahlen eines Schwerpunktversorgers im Verlauf der Corona-Pandemie in Mecklenburg-Vorpommern
}

\author{
Impact of COVID-19 Outbreak on Emergency Department \\ Admissions in a Specialized Hospital
}

Autoren

Mathias Stöwhas ${ }^{1,2}$, Hans Lippert ${ }^{3}$

Institute

1 Medizincontrolling, KMG Kliniken SE, Bad Wilsnack, Deutschland

2 Fakultät Gesundheitswissenschaften, MSB Medical School Berlin GmbH, Berlin, Deutschland

3 Klinik für Allgemein-, Viszeral-, Gefäß- und Transplantationschirurgie, Otto von Guericke Universitat Magdeburg, Magdeburg, Deutschland

\section{Schlüsselwörter}

Corona-Pandemie, Notaufnahmefallzahlen, Nutzung medizinscher Ressourcen, Pandemiemaßnahmen, Verhalten Bevölkerung

Key words Covid-19, social and behavioral epidemiology, emergency department, admission, reduction of cases

online-publikation $\quad 17.03 .2021$

\section{Bibliografie}

Gesundheitswesen 2021; 83: 265-273

DOI 10.1055/a-1320-0479

ISSN 2194-9379

(C) 2021. Thieme. All rights reserved.

Georg Thieme Verlag KG, Rüdigerstraße 14,

70469 Stuttgart, Germany

Korrespondenzadresse

Mathias Stöwhas

KMG Kliniken SE

Medizincontrolling

Badstraße 5-7

19336 Bad Wilsnack

Deutschland

m.stoewhas@kmg-kliniken.de

\section{ZUSAMMENFASSUNG}

Einleitung Als Reaktion auf die Corona-Pandemie fand im Frühjahr 2020 ein gesellschaftlicher Stillstand statt. Untersucht werden soll, wie diese Pandemie-Maßnahme auf die Be- völkerung eines ländlich geprägten, strukturschwachen Bundeslandes am Beispiel der Notaufnahmefallzahlen eines Schwerpunktversorgers gewirkt hat.

Methoden Abrufvon anonymisierten aggregierten Routinedaten und Auswertung der Phasen „Vergleich“ (03.07.201915.03.2020), Corona-Phase 1 “ (16.03.2020-06.05.2020) und „Corona-Phase 2“ (07.05.2020-31.07.2020).

Ergebnisse Auswertung von 24977 Patientendaten (2493 Patienten Corona-Phase 1/5106 Patienten Phase 2). $53 \%$ der Patienten sind älter als 50 Jahre, ca. $30 \%$ älter als 70 Jahre. Regulär suchen 67,6 Patienten die Notaufnahme je Tag auf. In der Corona-Phase 1 reduziert sich die Zahl um 19,7 Patienten/Tag; in der Phase 2 sind 8,2 Patienten/Tag weniger als in der Vergleichsgruppe - bei insgesamt unveränderter Altersstruktur. $56 \%$ der Patienten suchen eigenständig (Selbstvorstellung) die Notaufnahme auf, 24\% auf Veranlassung eines Vertragsarztes, $17 \%$ werden durch den Rettungsdienst (Notarzt, RTW) vorgestellt. Besonders auffällig ist der Fallzahlrückgang bei den Patienten, die sich selbst in der Notaufnahme vorstellen und vom Kassenarzt eingewiesen werden. Die Fallzahlen über den Rettungsdienst (NEF, RTW) sind relativ stabil - bzw. in der 2 . Corona-Phase anteigend (Notarzt $+7 \%$, RTW + 36\%). Das Hauptdiagnosespektrum der aus der Notaufnahme stationär aufgenommenen Patienten umfasst bei den Vertragsarzteinweisungen und Selbstvorsteller 375 bzw. 360 ICD-10-GM Dreistellergruppen. Hierbei reduzieren sich typische Notfallerkrankungen deutlich (Hirninfarkt (I63) -52\%, Herzinfarkt (I21) $-61 \%$, Cholelithiasis (K80) $-75 \%$ bei Selbstvorstellungen/ Vorhofflimmern (148) - 55\%, akute Bronchitis (J20) - 35\%, Gastroenteritis (A09) $-48 \%$ bei Vertragsarzteinweisungen - jeweils Corona-Phase 1 zu Vergleichsgruppe). Diese Fallzahlverluste sind überwiegend in der Corona-Phase 2 persistent (Hirninfarkt (I63) - 29\% bei Selbstvorstellern) und nur z. T. reversibel (Herzinfarkt (I21)+10\%).

Schlussfolgerung Eine Reduktion von Notfallkontakten, die nicht final durch den Pandemieverlauf erklärbar ist, v. a. der Selbstvorstellungen, Kassenarztvorstellungen fanden statt. Die Reduktion der Fallzahlen v. a. der überwiegend älteren Patienten, die selbstständig oder über den Kassenarzt vorgestellt wurden, überwog den Anteil des professionellen Rettungsdi- 
enstes deutlich. Es kann vermutet werden, dass PandemieMaßnahmen selbst zu diesem Effekt - trotz ausreichender medizinischer Ressourcen- geführt haben. „Infektionsangst“ als alleiniges Erklärungsmodell überzeugt nicht, da in Mecklenburg die Pandemie einen sehr milden, fallzahlschwachen Verlauf ohne Hotspot-Ereignisse in Krankenhäusern hatte. Untersuchungen zur Entscheidungsfindung der Bevölkerung bei Notfällen im weiteren Pandemieverlauf sind notwendig.

\section{ABSTRACT}

Background Extensive lockdown restrictions were implemented by the government to cope with the COVID-19 pandemic situation.

Objectives We investigated whether and how access to the emergency department (ED) changed during the COVID-19 outbreak compared to baseline parameter in a hospital in the county Mecklenburg in Germany.

Materials and methods Data on patients who accessed the ED in "Corona - Phase 1" (16.03.2020-06.05.2020) and "Co- rona - Phase 2" (07.05.2020-31.07.2020) were collected and compared with the "pre-Covid-era" (03.07.2019-15.03.2020). Results Data on 24,977 patients were evaluated; of these, 2493 patients in the Corona-Phase 1 and 5106 patients in the CoronaPhase 2 periods. Among these patients, $53 \%$ patients were older than $50,30 \%$ older than 70 years. Normally 67.6 patients/ day (p/d) visit the ED. This value decreased to $19.7 \mathrm{p} / \mathrm{d}$ in Phase 1 and $8.2 \mathrm{p} / \mathrm{d}$ in phase $2.24 \%$ of the patients were referred by the GP, $56 \%$ presented themselves and $17 \%$ by the rescue service. Wo observed a decline of stroke cases by $52 \%$, heart attacks by $61 \%$ and atrial fibrillation by $55 \%$ in Phase 1 . The decline of cases mainly persisted in phase 2 (e. g. stroke $29 \%$ ).

Conclusions Access to the ED decreased during the COVID-19 outbreak especially by patients who presented to the ED by themselves or referred by the GP - these were more than those brought into the ED by the rescue service. Based on literature and our own data, we suspect the lockdown restrictions could be responsible for the observed decrease of cases, too. In the context of the mild course of COVID-19 cases in Mecklenburg, these results are surprising. Further studies regarding whether and how the population used the ED should be performed.

\section{Einleitung}

Erstmals im Dezember 2019 in der Stadt Wuhan nachgewiesen, kam es in den folgenden Wochen zu einer weltweiten Ausbreitung des SARS-CoV-2-Virus [1, 2] Mitte Februar 2020 war das Virus dann in Europa und Deutschland angekommen und ein sprunghafter Anstieg der Fallzahlen begann [3]. Die Politik und Krankenhäuser reagierten mit der Bereitstellung von Behandlungsressourcen und der Absage planbarer Krankenhausbehandlungen [4, 5]. Das soziale Leben wurde durch Allgemeinverfügungen besonders ab dem 16. März zum Erliegen gebracht [6]. Aufgrund niedriger CoVid19-Fallzahlen konnten Lockerungen der Maßnahmen im Verlauf ermöglicht werden. [7]

Mehrere Arbeiten befassten sich in Folge mit den Veränderungen der vollstationären Fallzahl in den deutschen Krankenhäusern [8-10]. Der WIdO-Report konnte für den März und April 2020 im Vergleich Vorjahres-Zeitraums deutliche Fallzahlrückgänge von $39 \%$ nachweisen [8]. Die deutlichsten Fallzahlrückgänge konnten beim Arthrose-bedingten Hüftersatz ( $-79 \%$ ), bei Eingriffen zur Rekonstruktion an der Brust ( $-76 \%$ ) und am Darm ( $-70 \%)$ gesichert werden [8]. Überraschenderweise zeigte der WIdO-Report auch starke Rückgänge bei der Behandlung von Herzinfarkten ( - 31\%) und Schlaganfällen (-18\%) [8]. Ähnliche Fallzahlreduktionen in diesen Gruppen konnten aus eher universitär geprägten Notaufnahmen bestätigt werden [9].

Im Bundesland Mecklenburg-Vorpommern hatte im Verlauf der Corona-Pandemie bis zum 31.07.2020 stets sehr niedrige CoVid19-Fallzahlen sowie die niedrigste Sterblichkeit im Bundesvergleich beobachtet werden [11].

Es soll untersucht werden, wie sich die Fallzahlen von Patienten in einer Notaufnahme eines Schwerpunktversorgers im Bundesland Mecklenburg-Vorpommern im Zeitraum vom 16.03.2020 bis
31.07.2020 veränderten. Das betrachtete Krankenhaus Güstrow (regionales Traumazentrum, Chest Pain und Stroke Unit) versorgt ca. 22000 Patienten stationär und ca. 25000 Patienten ambulant pro Jahr, ist Standort eines Rettungshubschraubers und Notarzteinsatzfahrzeuges.

\section{Material und Methoden}

Ab Juli 2019 wurden Notaufnahmebehandlungen digital dokumentiert, so dass diese Routinedaten ohne Fallnummern/Patientenbezug aggregiert und so anonymisiert abgefragt wurden. Es werden die Art der Einweisung, der Wochentag, das Alter, die Dringlichkeitsstufe im MTS-System, sowie bei im Verlauf vollstationär aufgenommenen Fällen die Hauptdiagnose des Falles [12] abgerufen.

Drei Zeiträume werden verglichen:

1) „Vergleich“ - 03.07.2019-15.03.2020

2) „Corona - Phase 1“ - 16.03.2020-06.05.2020 („Lockdown“Phase in M-V)

3) „Corona - Phase 2“ - 07.05.2020-31.07.2020 (Beginn Lockerung)

Der Beginn des Lockdown um den 16.03.2020 wurde medial deutlich begleitet, ebenfalls der Beginn der ersten Lockerungsphase im MV-Plan. Diese beiden zeitlichen Zäsuren bilden die erste Corona-Phase ab, die hinsichtlich der Wirkung auf die Bevölkerung untersucht werden soll. Das Ende des Untersuchungszeitraumes beschreibt das Ende der Sommerferien in Mecklenburg.

Bei dieser Arbeit wurden aggregierter Daten über einen längeren Zeitraum ohne Fallnummernbezug und Einsicht in den Behandlungsunterlagen verwendet. Aus der Darstellung kann kein Personenbezug mehr konstruiert werden. 


\section{Ergebnisse}

Im gesamten Untersuchungszeitraum wurden 24977 Patienten behandelt, wobei 2493 Patienten auf die erste Corona-Phase und 5106 Patienten auf die zweite Corona-Phase entfallen. Die jeweiligen Phasen haben eine z.T. deutlich unterschiedliche Dauer, sodass rechnerisch die Fälle/Tag bestimmt werden, um die Phasen miteinander vergleichen zu können. Im Vergleichszeitraum wurde die Notaufnahme des Krankenhauses Güstrow rechnerisch von 67,6 Patienten je Tag aufgesucht ( $\triangleright$ Tab. 1).

In der Corona-Phase 1 reduzierte sich die Zahl der Patienten in der Notaufnahme um fast $30 \%$. In der Corona-Phase 2 erhöhte sich die Zahl der Notaufnahmepatienten wieder, lag aber dennoch $12 \%$ unter dem Vergleichszeitraum. Die Altersstruktur der Patienten veränderte sich kaum ( $\triangleright$ Tab. 1).

Innerhalb der Notaufnahme werden die Fälle verschieden Fachrichtungen zugeordnet ( $\triangleright$ Tab. 2). Der Rückgang der Patientenzahlen verteilt sich sehr unterschiedlich über die Fachrichtungen. In den Fallzahl-starken Fachrichtungen (Chirurgie, Innere Medizin) war der Rückgang der Patienten in der Chirurgie doppelt so groß, wie in der Inneren Medizin. Beide Fachrichtungen hatten in der 2. Corona-Phase dann gemeinsam $9 \%$ weniger Patienten als in der Vergleichsphase.

Die Fachrichtung Pädiatrie verlor $60 \%$ ihrer Patienten, die Gynäkologie fast $50 \%$, die Neurologie fast $40 \%$, wobei der Effekt des Fallzahlrückgangs in der Pädiatrie auch in der 2. Corona-Phase deutlicher anhält als bei allen anderen Abteilungen. Die Urologie hingegen war nahezu konstant im Fallzahlaufkommen.

Die meisten Patienten suchten die Notaufnahme selbstständig (ohne Einweisung), auf Veranlassung eines Vertragsarztes oder unter Nutzung des Rettungsdienstes (RTW, Notarzt) auf. Die ersten beiden Gruppen zeigte die deutlichste absolute und relative Reduktion. In der ersten Corona-Phase änderten sich die Fallzahlen im Bereich des Rettungsdienstes kaum, es kam jedoch in den anderen beiden Bereichen zu einer deutlichen -und auch in der 2.Corona-Phase nicht vollständig rekompensierten- Reduktion der Patientenzahlen ( $\triangleright$ Tab. 3). Patienten, die über den Rettungsdienst (RTW, Notarzt) eingewiesen wurden, waren in der Anzahl relativ konstant bzw. im Falle der RTW-Einweisungen in der 2. CoronaPhase sogar ansteigend.

Die zahlenmäßig größte Patientengruppen (Selbstvorsteller, Vertragsarzteinweisungen) zeigten deutliche Veränderungen und wurden vertieft analysiert. Aus Datenschutzgründen konnte die
Patientenakte nicht genutzt wurden, sodass die Angabe einer Diagnose für ambulanten Notaufnahmepatienten nicht möglich. Für die Patienten, die aus der Notaufnahme stationär aufgenommen wurden, konnte die Hauptdiagnose des Falles gemäß der für Deutschland gültigen Internationalen Klassifikation der Krankheiten (ICD-10-GM 2020) und in Anwendung der Deutschen Kodierrichtlinien [12] bestimmt werden. Ein ICD-10-GM 2020 Code beinhaltet bis zu 5 Stellen (Beispiel: 110.00 - Benigne essentielle Hypertonie ohne Angabe einer hypertensiven Krise). Zur besseren Übersicht wurden die kodierten Hauptdiagnosen in Form von Dreisteller-Codes (im obigen Beispiel I10) zusammengefasst. Den beiden betrachten Gruppen „Vertragsarzteinweisungen“ und „Selbstvorsteller“ konnten so 375 bzw. 360 unterschiedlicher ICD10-GM - Dreistellergruppen zugeordnet werden. In der weiteren Darstellung wurden zur Vereinfachung die ICD-Dreisteller-Codes ausgewählt, die die deutlichsten absoluten oder relativen Veränderungen zeigten.

In > Tab. $\mathbf{4}$ und $\mathbf{5}$ wurden jeweils die TOP-20-ICD-Dreisstellergruppen ausgewählt, die in der Vergleichsgruppe die höchsten Fallzahlen auswiesen und deren Veränderungen in der Corona-Pandemie dargestellt. (die komplette Übersicht findet sich im Anhang)

Es zeigt sich, dass sich bspw. vor allem in der ersten CoronaPhase $52 \%$ weniger Hirninfarkte als Selbstvorsteller in die Notaufnahme begaben. Auch der Anteil der dann stationär aufgenommenen akuten Bronchitis, COPD und der Pneumonie verminderte sich um ca. $80 \%$. Patienten mit einem Myocardinfarkt oder einer Herzinsuffizienz nahmen um 30-60\% ab. Patienten mit einer Depression fanden als Selbstvorsteller keinen Weg mehr in die Notaufnahme. Dieser geschilderte Trend zeigt sich auch bei den Patientengruppen, die über den Vertragsarzt eingewiesen wurden.

\section{Diskussion}

Die vorliegende Arbeit beschreibt einen Rückgang von Patienten in der Notaufnahme eines Schwerpunktversorgers im Land Mecklenburg-Vorpommern um ca. 30 \%. in der Zeit vom 16.03.202031.07.2020.

In der Abb. 1 soll der Weg eines Patienten von der Wahrnehmung eines Krankheitsbildes bis zur Vorstellung in der Notaufnahme vereinfacht dargestellt werden. An diesem Schaubild lassen sich möglich Einflussfaktoren, die den beobachteten Effekt der Fallzahlreduktion begründen könnten, ableiten. Krankheits-

- Tab. 1 Fälle und Alterskohorten der Notaufnahmepatienten.

\begin{tabular}{|c|c|c|c|c|c|c|c|c|c|}
\hline & \multicolumn{3}{|c|}{ Vergleichszeitraum } & \multicolumn{3}{|c|}{ Corona - Phase 1} & \multicolumn{3}{|c|}{ Corona - Phase 2} \\
\hline Tage je Zeitraum & \multicolumn{3}{|c|}{257} & \multicolumn{3}{|c|}{52} & \multicolumn{3}{|c|}{86} \\
\hline Fälle Notaufnahme & \multicolumn{3}{|c|}{17378} & \multicolumn{3}{|c|}{2493} & \multicolumn{3}{|c|}{5106} \\
\hline Fälle/Tag & \multicolumn{3}{|c|}{67,6} & \multicolumn{3}{|c|}{47,9} & \multicolumn{3}{|c|}{59,4} \\
\hline Alterskohorte & relativ & absolut & Fälle/Tag & relativ & absolut & Fälle/Tag & relativ & absolut & Fälle/Tag \\
\hline kleiner 10 Jahre & $12 \%$ & 2100 & 8,2 & $9 \%$ & 228 & 4,4 & $10 \%$ & 489 & 5,7 \\
\hline 10-17 Jahre & $7 \%$ & 1162 & 4,5 & $4 \%$ & 109 & 2,1 & $5 \%$ & 255 & 3,0 \\
\hline 18-49 Jahre & $28 \%$ & 4859 & 18,9 & $28 \%$ & 686 & 13,2 & $26 \%$ & 1328 & 15,4 \\
\hline 50-69 Jahre & $25 \%$ & 4399 & 17,1 & $27 \%$ & 673 & 12,9 & $29 \%$ & 1476 & 17,2 \\
\hline 70-89 Jahre & $26 \%$ & 4477 & 17,4 & $29 \%$ & 733 & 14,1 & $28 \%$ & 1445 & 16,8 \\
\hline größer 90 Jahre & $2 \%$ & 382 & 1,5 & $3 \%$ & 64 & 1,2 & $3 \%$ & 132 & 1,5 \\
\hline
\end{tabular}



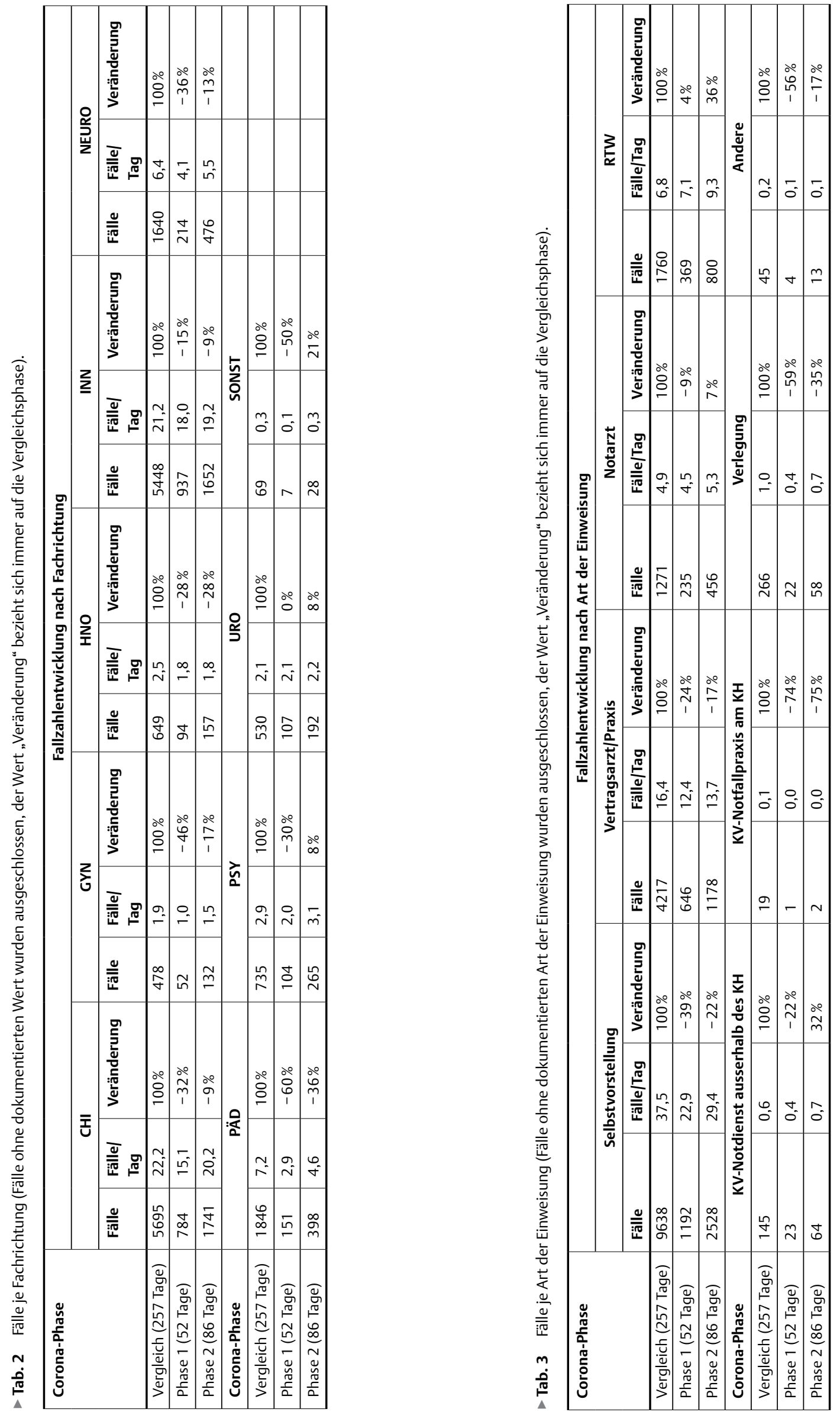
> Tab. 4 Selbstvorstellung in Notaufnahme (TOP 20 der Vergleichsgruppe).

\begin{tabular}{|c|c|c|c|c|c|c|c|c|}
\hline \multirow[t]{2}{*}{ Selbstvorstellung } & \multicolumn{2}{|c|}{ Vergleichsgruppe (257 Tage) } & \multicolumn{3}{|c|}{ Corona-Phase 1 (52 Tage) } & \multicolumn{3}{|c|}{ Corona-Phase 2 (86 Tage) } \\
\hline & Fälle & Fälle/Tag & Fälle & $\begin{array}{l}\text { Fälle| } \\
\text { Tag }\end{array}$ & $\begin{array}{l}\text { Veränderung } \\
\text { zu Vergleich }\end{array}$ & Fälle & $\begin{array}{l}\text { Fälle/ } \\
\text { Tag }\end{array}$ & $\begin{array}{l}\text { Veränderung } \\
\text { zu Vergleich }\end{array}$ \\
\hline S06 Intrakranielle Verletzung & 120 & 0,47 & 20 & 0,38 & $-18 \%$ & 32 & 0,37 & $-20 \%$ \\
\hline I63 Hirninfarkt & 72 & 0,28 & 7 & 0,13 & $-52 \%$ & 17 & 0,20 & $-29 \%$ \\
\hline $\begin{array}{l}\text { F10 Psychische und Verhaltens- } \\
\text { störungen durch Alkohol }\end{array}$ & 67 & 0,26 & 14 & 0,27 & $3 \%$ & 14 & 0,16 & $-38 \%$ \\
\hline $\begin{array}{l}\text { N39 Sonstige Krankheiten des } \\
\text { Harnsystems }\end{array}$ & 62 & 0,24 & 8 & 0,15 & $-36 \%$ & 16 & 0,19 & $-23 \%$ \\
\hline K80 Cholelithiasis & 60 & 0,23 & 3 & 0,06 & $-75 \%$ & 14 & 0,16 & $-30 \%$ \\
\hline $\begin{array}{l}\text { B34 Viruskrankheit nicht näher } \\
\text { bezeichneter Lokalisation }\end{array}$ & 60 & 0,23 & 3 & 0,06 & $-75 \%$ & 10 & 0,12 & $-50 \%$ \\
\hline J20 Akute Bronchitis & 53 & 0,21 & 2 & 0,04 & $-81 \%$ & 6 & 0,07 & $-66 \%$ \\
\hline I50 Herzinsuffizienz & 51 & 0,20 & 7 & 0,13 & $-32 \%$ & 12 & 0,14 & $-30 \%$ \\
\hline $\begin{array}{l}\text { A09 Sonstige Gastroenteritis und } \\
\text { Kolitis }\end{array}$ & 48 & 0,19 & 3 & 0,06 & $-69 \%$ & 8 & 0,09 & $-50 \%$ \\
\hline K29 Gastritis und Duodenitis & 47 & 0,18 & 6 & 0,12 & $-37 \%$ & 26 & 0,30 & $65 \%$ \\
\hline R10 Bauch- und Beckenschmerzen & 40 & 0,16 & 4 & 0,08 & $-51 \%$ & 8 & 0,09 & $-40 \%$ \\
\hline $\begin{array}{l}110 \text { Essentielle (primäre) } \\
\text { Hypertonie }\end{array}$ & 39 & 0,15 & 4 & 0,08 & $-49 \%$ & 9 & 0,10 & $-31 \%$ \\
\hline I21 Akuter Myokardinfarkt & 38 & 0,15 & 3 & 0,06 & $-61 \%$ & 14 & 0,16 & $10 \%$ \\
\hline $\begin{array}{l}\text { J44 Sonstige chronische } \\
\text { obstruktive Lungenkrankheit }\end{array}$ & 36 & 0,14 & 1 & 0,02 & $-86 \%$ & 3 & 0,03 & $-75 \%$ \\
\hline $\begin{array}{l}148 \text { Vorhofflimmern und } \\
\text { Vorhofflattern }\end{array}$ & 36 & 0,14 & 9 & 0,17 & $24 \%$ & 10 & 0,12 & $-17 \%$ \\
\hline $\begin{array}{l}\text { H81 Störungen der Vestibular- } \\
\text { funktion }\end{array}$ & 30 & 0,12 & 3 & 0,06 & $-51 \%$ & 8 & 0,09 & $-20 \%$ \\
\hline $\begin{array}{l}\text { J18 Pneumonie, Erreger nicht } \\
\text { näher bezeichnet }\end{array}$ & 29 & 0,11 & 1 & 0,02 & $-83 \%$ & 4 & 0,05 & $-59 \%$ \\
\hline $\begin{array}{l}\text { N13 Obstruktive Uropathie und } \\
\text { Refluxuropathie }\end{array}$ & 29 & 0,11 & 6 & 0,12 & $2 \%$ & 6 & 0,07 & $-38 \%$ \\
\hline $\begin{array}{l}\text { S39 Sonstige Verletzungen des } \\
\text { Abdomens, Lumbosakralgegend \& } \\
\text { Beckens }\end{array}$ & 25 & 0,10 & 1 & 0,02 & $-80 \%$ & 8 & 0,09 & $-4 \%$ \\
\hline $\begin{array}{l}\text { A08 Virusbedingte und sonstige } \\
\text { näher bezeichnete Darminfek- } \\
\text { tionen }\end{array}$ & 25 & 0,10 & 0 & 0,00 & $-100 \%$ & 2 & 0,02 & $-76 \%$ \\
\hline
\end{tabular}

bilder treten in manchen Fällen saisonal oder in Folge bestimmter Ereignisse auf (Grippe-Saison, traumatologische Krankheitsbilder im Winter, kardiologische Krankheitsbilder in Folge von Hitzeperioden) $[13,14]$. Extremwetterlagen sind in den betrachteten Zeiträumen nicht bekannt.

Die meisten Patienten der Notaufnahme stammen aus 3 Quellen: Selbstvorstellungen, Einweisungen durch den Vertragsarzt und durch den Rettungsdienst (Notarzt, RTW). Die Selbstvorstellungen reduzierten sich in betrachteten Zeitraum in den wesentlichen Diagnosegruppen ubiquitär und deutlich. Einweisungen durch den Vertragsarzt waren in Summe auch rückläufig, jedoch zeigten sich in einzelnen Diagnosegruppen v. a. in der LockdownPhase konstante (N39 - Harnwegsinfekte, H81 - Schwindel), ansteigende (K29 - Gastritis/Duodenitis, K80 - Cholelithiasis, E11 Diabetes mellitus) oder komplett zu den Selbstvorstellungen divergente (I63 - Hirninfarkt) Fallzahlentwicklungen. Die Fallzahlen des Rettungsdienstes waren relativ konstant bis leicht ansteigend in der Phase 2. Wir wissen nicht, wie viele Patienten sich initial in den Praxen bzw. bei der Leitstelle gemeldet haben, sodass die beobachteten Effekte nicht zwingend für eine in der Literatur erhöhten Rate des Vor-Ort-Verbleibens [10,15] sprechen müssen. Für die weitere Analyse des vertragsärztlichen Bereiches wären Daten der Kassenärztlichen Vereinigung hinsichtlich Scheinzahlen, Diagnosezahlen und Auswertung von erbrachten Leistungen i.S. der abgerechneten EBM-Positionen hilfreich.

Hinsichtlich der Krankenhäuser ist die Wirkung der Freihaltepauschale von 560 Euro zur Kompensation von Erlösausfällen ist in der Literatur in Diskussion [16]. Jedoch entfaltet dieser Komplex bei den vorliegenden Daten keine Wirkung, da nicht planbare Operationen und Behandlungen ausgewertet werden, sondern die nicht steuerbare Zuweisung/Vorstellung von Patienten in der Notaufnahme. 
- Tab. 5 Einweisung durch Vertragsarzt in die Notaufnahme (TOP 20 der Vergleichsgruppe).

\begin{tabular}{|c|c|c|c|c|c|c|c|c|}
\hline \multirow[t]{2}{*}{ Vertragsarzteinweisung } & \multicolumn{2}{|c|}{ Vergleichsgruppe (257 Tage) } & \multicolumn{3}{|c|}{ Corona-Phase 1 (52 Tage) } & \multicolumn{3}{|c|}{ Corona-Phase 2 (86 Tage) } \\
\hline & Fälle & Fälle/Tag & Fälle & $\begin{array}{l}\text { Fälle/ } \\
\text { Tag }\end{array}$ & $\begin{array}{l}\text { Veränderung } \\
\text { zu Vergleich }\end{array}$ & Fälle & $\begin{array}{l}\text { Fälle/ } \\
\text { Tag }\end{array}$ & $\begin{array}{l}\text { Veränderung } \\
\text { zu Vergleich }\end{array}$ \\
\hline 150 Herzinsuffizienz & 140 & 0,54 & 25 & 0,48 & $-12 \%$ & 41 & 0,48 & $-12 \%$ \\
\hline K29 Gastritis und Duodenitis & 83 & 0,32 & 23 & 0,44 & $37 \%$ & 21 & 0,24 & $-24 \%$ \\
\hline $\begin{array}{l}\text { I48 Vorhofflimmern und Vorhofflat- } \\
\text { tern }\end{array}$ & 77 & 0,30 & 7 & 0,13 & $-55 \%$ & 24 & 0,28 & $-7 \%$ \\
\hline $\begin{array}{l}\text { N39 Sonstige Krankheiten des } \\
\text { Harnsystems }\end{array}$ & 68 & 0,26 & 13 & 0,25 & $-6 \%$ & 35 & 0,41 & $54 \%$ \\
\hline J20 Akute Bronchitis & 53 & 0,21 & 7 & 0,13 & $-35 \%$ & 6 & 0,07 & $-66 \%$ \\
\hline $\begin{array}{l}\text { J18 Pneumonie, Erreger nicht näher } \\
\text { bezeichnet }\end{array}$ & 50 & 0,19 & 8 & 0,15 & $-21 \%$ & 3 & 0,03 & $-82 \%$ \\
\hline 170 Atherosklerose & 49 & 0,19 & 4 & 0,08 & $-60 \%$ & 10 & 0,12 & $-39 \%$ \\
\hline K80 Cholelithiasis & 47 & 0,18 & 12 & 0,23 & $26 \%$ & 21 & 0,24 & $34 \%$ \\
\hline I20 Angina pectoris & 42 & 0,16 & 5 & 0,10 & $-41 \%$ & 10 & 0,12 & $-29 \%$ \\
\hline 163 Hirninfarkt & 39 & 0,15 & 9 & 0,17 & $14 \%$ & 13 & 0,15 & $0 \%$ \\
\hline $\begin{array}{l}\text { B34 Viruskrankheit nicht näher } \\
\text { bezeichneter Lokalisation }\end{array}$ & 38 & 0,15 & 3 & 0,06 & $-61 \%$ & 4 & 0,05 & $-69 \%$ \\
\hline $\begin{array}{l}\text { A09 Sonstige und nicht näher } \\
\text { bezeichnete Gastroenteritis und } \\
\text { Kolitis infektiösen und nicht näher } \\
\text { bezeichneten Ursprungs }\end{array}$ & 38 & 0,15 & 4 & 0,08 & $-48 \%$ & 12 & 0,14 & $-6 \%$ \\
\hline E11 Diabetes mellitus, Typ 2 & 37 & 0,14 & 11 & 0,21 & $47 \%$ & 6 & 0,07 & $-52 \%$ \\
\hline K57 Divertikulose des Darmes & 36 & 0,14 & 3 & 0,06 & $-59 \%$ & 5 & 0,06 & $-58 \%$ \\
\hline $\begin{array}{l}\text { J44 Sonstige chronische obstruktive } \\
\text { Lungenkrankheit }\end{array}$ & 35 & 0,14 & 8 & 0,15 & $13 \%$ & 6 & 0,07 & $-49 \%$ \\
\hline R10 Bauch- und Beckenschmerzen & 34 & 0,13 & 3 & 0,06 & $-56 \%$ & 11 & 0,13 & $-3 \%$ \\
\hline I10 Essentielle (primäre) Hypertonie & 34 & 0,13 & 5 & 0,10 & $-27 \%$ & 7 & 0,08 & $-38 \%$ \\
\hline R07 Hals- und Brustschmerzen & 32 & 0,12 & 5 & 0,10 & $-23 \%$ & 12 & 0,14 & $12 \%$ \\
\hline $\begin{array}{l}\text { H81 Störungen der Vestibularfunk- } \\
\text { tion }\end{array}$ & 31 & 0,12 & 7 & 0,13 & $12 \%$ & 5 & 0,06 & $-52 \%$ \\
\hline $\begin{array}{l}\text { N13 Obstruktive Uropathie und } \\
\text { Refluxuropathie }\end{array}$ & 29 & 0,11 & 3 & 0,06 & $-49 \%$ & 11 & 0,13 & $13 \%$ \\
\hline
\end{tabular}

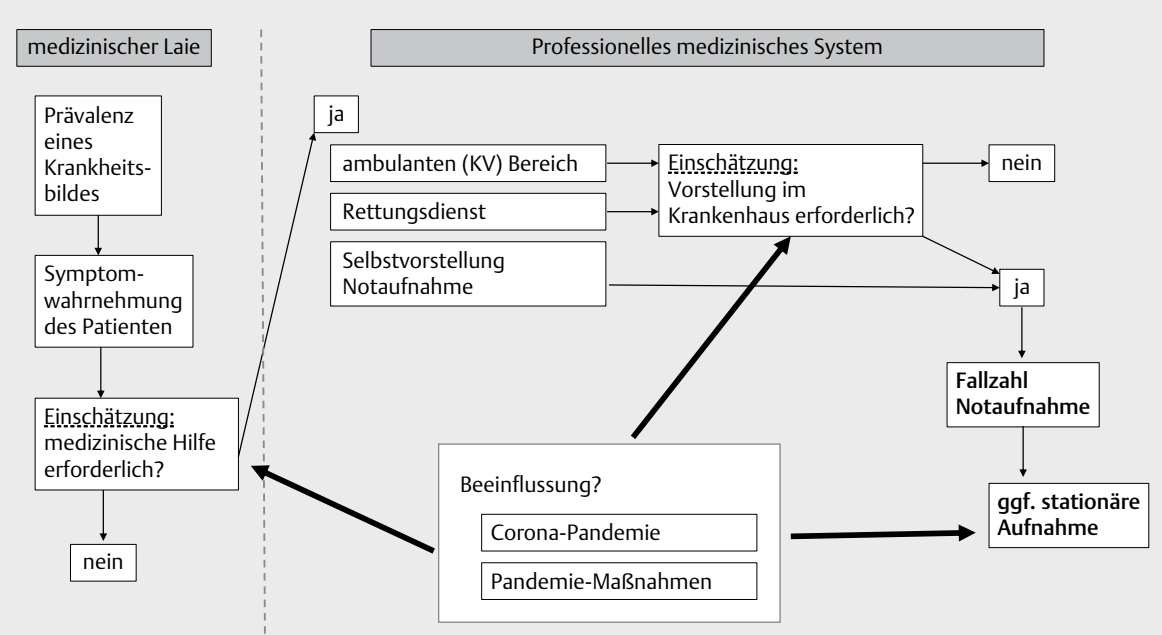

- Abb 1 Vom Krankheitsbild zur stationären Aufnahme (eigene Darstellung). 
Betrachtet man bestimmte Krankheitsbilder genauer, lassen sich Verknüpfung zur Pandemie-Situation finden und pathophysiologisch erklärbare Inzidenzreduktionen herleiten.

Herzinfarkte entwickeln sich auch in Folge von körperlicher Anstrengung [17, 18]. Die Pandemie-Maßnahmen (Absage Sportveranstaltung, Einschränkung der Mobilität) könnten so zu einer realen Reduktion der Inzidenz geführt haben. Die Absage von Veranstaltungen führte zu weniger Verkehr [19]. Für den Zeitraum März-Juni kam es in Deutschland zu 26\% weniger Verkehrsunfällen mit 21,4\% weniger Personenschäden [43]. Es erscheint plausibel, dass Notaufnahmeanlässe wie Unfälle und Verletzungen abnehmen [20]. Die Sorge vor einer Infektion hat in der Literatur zu einer verzögerten Inanspruchnahme bei Herzinfarkten geführt [21, 22]. Pandemie-Maßnahme können bei Menschen Stress/seelische Belastung auslösen, was in der Literatur wieder mit einem vermehrten Auftreten von Herzinfarkten und Schlaganfällen verbunden ist [23]. Die Auswertung von Notaufnahmedaten [9] von mehrheitlich Universitätsstandorten konnte übereinstimmend mit unseren Daten den Trend der Reduktion z. B. der Schlaganfälle ( - 52 \%) und TIA ( $-100 \%)$ finden. Überraschenderweise fanden sich bei den Patienten, die durch den Vertragsarzt vorgestellt wurden eher stabile Zahlen ( $+14 \%$ ) beim Hirninfarkt, jedoch auch $-36 \%$ bei der TIA. Passend zu den Befunden in der Literatur sahen auch wir eine deutliche Reduktion kardiologischer Patienten (Selbstvorsteller: Infarkt - 61\%; Vertragsarzt - 36\%). Äußerst deutlich sahen wir auch eine Reduktion der Patienten aus dem psychiatrischen Gebiet - Patienten mit depressiven Störungsbildern fanden kaum noch den Weg in die Notaufnahme. Eine Reduktion der psychiatrischen Fälle über 50 \% ist auch in Portugal währen der Corona-Pandemie beobachtet worden [24]. Psychiatrische Patienten hatten Angst vor einer Infektion oder glaubten (fehlerhaft), dass Krankenhäuser nur noch Corona-Patienten behandeln und/oder dass ihre Beschwerden nicht schlimm genug seien, um als ein Notfall zu gelten [25].

Krankheitsbilder, die in ihrem initialen klinischen Bild einer SARS-CoV-2-Infektion ähnlich sind (J20 - Akute Bronchitis, J18 Pneumonie und J44 - COPD) gehen bei den Selbsteinweisungen um $80 \%$ in der Phase 1 zurück. Bei den Vertragsarzteinweisungen sind die Rückgänge weniger deutlich (J20-35\%,/J18-21\%) bzw. divergent $(C O P D+13 \%)$ in Phase 1. Fallzahlrückgänge im Pandemieverlauf (COPD - 39\%) werden auch aus Italien [44] bzw. (COPD -48\% und Pneumonie ( $-45 \%$ ) aus den USA beschrieben [45]. Ein Erklärungsmodell mag -partiell- das Tragen eines Mund-NasenSchutzes in Kombination mit der sozialen Isolation sein. Der deutliche Rückgang der Selbsteinweisungen ist vielleicht auch der Angst der Patienten geschuldet, als Verdachtsfall isoliert zu werden.

Eine in jedem Fall finale medizinisch konsistente Begründung der beobachteten Fallzahlreduktion über pathophysiologische Überlegungen gelingt so nicht.

Kehrt man so zur $\triangleright$ Abb. 1 zurück, existieren für die Bewertung eines gesundheitlichen Zustandes aus Sicht eines medizinischen Laien verschiedene Modelle [26, 27]. Egal unter welcher Rationale die Bewertung stattfindet, entscheidet der Mensch (potenzielle Patient), ob er in das professionelle medizinische System eintreten möchte und auf welchem Weg (Hausarzt, Facharzt, Rettungsdienst, Selbstvorstellung in der Notaufnahme). Es mag insofern überraschen, dass Patienten mit unstreitigen Notfallindikationen (Schlaganfall, Herzinfarkt, Angina pectoris) als Selbstvorsteller die No- taufnahme betreten. Die Validität der subjektive (Laien-)Einschätzung der Gesundheit wird diskutiert [28].

Die bisherigen Punkte (Gesundheitskonzepte/Wahrnehmung von Krankheit/Bereitschaft ärztliche Hilfe in Anspruch zu nehmen) sind immanent in der Bevölkerung vertreten und wirken als „Hintergrundrauschen “ in der Vergleichsgruppe sicher mit. Lampert et al. fanden Unterschiede in der Lebenserwartung, Arbeitsunfähigkeitstagen und anderen Indikatoren auf Ebene von Kreisen und kreisfreien Städten [29] , so dass die eher „abwartende Haltung“ bevor medizinische Hilfe aufgesucht wird, vielleicht eine lokale Eigenheit ist. Neu ist jedoch die Corona-Pandemie hinzugekommen. Aus der SARS-Epidemie 2003 ist bekannt, dass die Fallzahlen der Notaufnahmen um $51,6 \%$ eingebrochen sind und sich dieses sowohl im pädiatrischen, traumatologischen und nicht-traumatologischen Bereich gezeigt hat. Dieser Effekt war noch 3 Monate nach dem Ende der Pandemie sichtbar [20]. Möglicherweise war hier die Angst vor einer Ansteckung [30] oder in den Medien berichtet Ausbrüche des Corona-Virus [31] in Krankenhäuser eine Ursache für die reduzierte Fallzahl in der Notaufnahme. Patienten mit -subjektiv erlebten- leichten Beschwerden haben nicht oder verspätet Kontakt mit dem Gesundheitssystem gesucht, weil sie eine Überlastung desselben vermuten oder ihre Beschwerden bagatellisieren [32].

Der Nationale Pandemieplan in Deutschland beschreibt Phasen und Ziele der Pandemiebekämpfung: Containment (Eindämmungsstrategie), Protection (Schutz vulnerabler Gruppen), Mitigation (Folgenminderung) und Recovery (Erholung) [47]. In Bezug auf das Containment wurden Allgemeinverfügungen erlassen [6, 34] , die das öffentliche Leben in der ersten Corona-Phase nachhaltig reduzierten (strenge Kontakt-/Besuchsbeschränkungen auch im Krankenhaus, Schließung von Geschäften, Absage von Veranstaltungen). Im Land Mecklenburg-Vorpommern galt zudem das Verbot als Nicht-Bewohner das Land zu betreten $[19,35,36]$. Heudorf stellt in ihrem Artikel dar, dass Erfahrung aus früheren Pandemien zeigen: „dass das Containment ab einer gewissen Ausbreitung eines neuartigen leichtübertragbaren Virus nicht mehr funktionieren kann, insbesondere, wenn das Virus viele asymptomatische und leichte Infektionen verursacht und die Infizierten unerkannt zu Überträgern werden “ [47]. Holst beschreibt in seiner Arbeit die besonderen Herausforderungen an Public Health im Kontext der Corona-Pandemie. Hier diskutiert er, dass für eine Reihe der o.g. Pandemie-Maßnahmen eine Abwägung der negativen Effekte in Relation zu den positiven (gewünschten) Wirkungen nicht ausreichend erfolgt ist [46].

Denkbar ist so, dass die Pandemie-Maßnahmen der Politik und der Krankenhäuser zu einer Reduktion der Fallzahlen in der Notaufnahme geführt haben. Denkbar ist, dass ältere Personen erst durch Angehörige oder Dritte bei Kontakten motiviert werden, medizinische Hilfe aufzusuchen - durch Kontaktbeschränkungen und/oder Einreiseverbote fehlen diese Anreize [15]. Hierfür mag die Normalisierung der Fallzahlen der Alterskohorte $>50$ Jahre in der Phase 2 ein Indiz sein ( $>$ Tab. 1). Andere Arbeitsgruppen fanden beobachtet auch zum Teil divergente Veränderungen in den Altersgruppen $[9,10]$. Auch denkbar ist, dass Patienten durch die ausgesprochenen Besuchsverbote in den Krankenhäusern abgehalten wurden, diese aufzusuchen [37-39].

Für die hier dargestellten Fallzahlen muss limitierend festgestellt werden, dass diese nur die Zahlen eines Hauses zeigen und in anderen Häusern divergieren können. Hier sei auf die unterschiedli- 
che Inanspruchnahme des Notarztes verwiesen [10]. Eine Verschiebung von Fällen in andere Krankenhäuser im Flächenland Mecklenburg-Vorpommern ist bei Notfällen eher unwahrscheinlich - aber nicht auszuschließen.

\section{FAZIT}

Im zeitlichen Kontext zur Corona-Pandemie kam es zu einer deutlichen Reduktion von Notfallpatienten und stationären Aufnahmen. Dieser Befund spiegelt sich in nationalen und internationalen Arbeiten wieder - obgleich sich das konkrete quantitative Ausmaß auf Diagnoseebene im Detail in den Arbeiten unterscheidet. Eine stringente, umfassende, pathophysiologisch-rational plausible Begründung der beobachteten Effekte gelingt nicht. Generalisiert man die gezeigten Daten auf das Land Mecklenburg-Vorpommern oder die Bundesrepublik Deutschland, würde es bedeuten, dass es in verschiedenen medizinischen Bereichen zu einer faktischen Unterversorgung gekommen ist - dieses nicht, weil medizinische Ressourcen nicht zur Verfügung standen, sondern weil Patienten das Gesundheitssystem nicht mehr gleichen Ausmaß genutzt haben, wie vor der Pandemie. Dieser Effekt ist auch aus dem Bereich der Onkologie zu verzeichnen [40-42]. Die Relevanz dieser Ableitung sei durch die Tatsache unterstrichen, dass es in MecklenburgVorpommern im betrachteten Zeitraum die niedrigsten Fallzahlen und die niedrigste Sterblichkeit in Deutschland in der Corona-Pandemie gab [11,33]. Es kann vermutet werden, dass (irrationale) Parameter wie „Angst und Sorge“, die nicht objektiv durch z. B. Inzidenzwerte oder Sterblichkeit getriggert werden oder auch Reaktionen auf die Pandemie-Maßnahmen selbst für die gezeigten Effekte in größerem Umfang ursächlich sein können. Hier sollte weitere Forschung stattfinden, um bei nachfolgenden Pandemie-Phasen oder einem neuen Erreger hoffentlich weniger „Nicht Pandemie-bedingte“ Verluste akzeptieren zu müssen.

\section{Interessenkonflikt}

M. Stöwhas ist als Angestellter für die KMG Kliniken SE tätig zu der auch das untersuchte Krankenhaus gehört. H. Lippert gibt an, dass kein Interessenkonflikt besteht.

\section{Literatur}

[1] Zhou P, Yang X-L, Wang X-G et al. A pneumonia outbreak associated with a new coronavirus of probable bat origin. Nature 579: 270-273

[2] Müller O, Neuhann F, Razum O. Epidemiologie und Kontrollmaßnahmen bei COVID-19. Dtsch Med Wochenschr 2020; 145: 670-674

[3] Schilling J, Diercke M, Altmann D et al. Vorläufige Bewertung der Krankheitsschwere von COVID-19 in Deutschland basierend auf übermittelten Fällen gemäß Infektionsschutzgesetz. Robert Koch-Institut. Epidemiologisches Bulletin 17/: 2020; 3-9
[4] Ramshorn-Zimmer A, Pin M, Hartwig T et al. Coronapandemie: Rolle der Zentralen Notaufnahme. Dtsch Arztebl 2020; 2020(117, A-1040/B-880)

[5] Möckel M, Bachmann U, Behringer W et al. How emergency departments prepare for virus disease outbreaks like COVID-19. Eur ] Emerg Med 2020; 27: 161-162

[6] Landesregierung Mecklenburg Vorpommern: Verordnung der Landesregierung über Maßnahmen zur Bekämpfung der Ausbreitung des neuartigen Coronavirus SARS-CoV-2 in Mecklenburg Vorpommern. Gesetz- und Verordnungsblatt Mecklenburg Vorpommern; 2020. https://www.regierung-mv.de/static/Regierungsportal/ Ministerium \%20f\%C3\%BCr\%20Wirtschaft \%2C\%20Arbeit \%20und \%20 Gesundheit/Dateien/Downloads/GVOBI \%20Nr\%206\%20vom \%20 18. \%20M \%C3\%A4rz \%202020\%20-\%20Corona.pdf (zuletzt aufgesucht am 30.09.20)

[7] Landesregierung Mecklenburg Vorpommern: Verordnung der Landesregierung zur weiteren schrittweisen Lockerung der coronabedingten Einschränkungen des öffentlichen Lebens in MecklenburgVorpommern 2020. https://www.regierung-mv.de/static/Regierungsportal/Portalredaktion/Inhalte/Corona/Corona-Verordnung.pdf (zuletzt aufgesucht am 30.09.20)

[8] Günster C, Drogan D, Hentschker C et al. WIdO-Report: Entwicklung der Krankenhausfallzahlen während des Coronavirus-Lockdowns. Wissenschaftliches Institut der AOK. 2020. https://www.aok-bv.de/ imperia/md/aokbv/hintergrund/dossier/krankenhaus/wido-report_fzentwicklung_lockdown.pdf (zuletzt aufgesucht am 30.09.20)

[9] Slagman A, Behringer W, Greiner F et al. Medizinische Notfälle während der COVID-19-Pandemie. Dtsch Arztebl Int 2020; 117 : 545-552

[10] Deutscher Ärzteverlag GmbH Redaktion Deutsches Ärzteblatt: Notaufnahme während der Coronapandemie: Weniger Non-COVID19-Notfälle. Dtsch Arztebl 2020; 117: A-1201. B-1016

[11] RKI: Täglicher Lagebericht des RKI zur Coronavirus-Krankheit-2019 (COVID-19): 31.07.2020 - AKTUALISIERTER STAND FÜR DEUTSCHLAND 2020. https://www.rki.de/DE/Content/InfAZ/N/ Neuartiges_Coronavirus/Situationsberichte/2020-07-31-de.pdf?__ blob = publicationFile (zuletzt aufgerufen am 30.09.20)

[12] Institut für das Entgeltsystem im Krankenhaus: Deutsche Kodierrichtlinien: Allgemeine und Spezielle Kodierrichtlinien für die Verschlüsselung von Krankheiten und Prozeduren 2020. https:// www.g-drg.de/G-DRG-System_2020/Kodierrichtlinien/Deutsche_ Kodierrichtlinien_2020 (zuletzt aufgesucht am 30.09.20)

[13] Huang S-J, Lin L-L, Chen L-C et al. Prevalence of airway hyperresponsiveness and its seasonal variation in children with asthma. Pediatr Neonatol 2018; 59: 561-566

[14] Kurlansik SL, Ibay AD. Seasonal affective disorder. Am Fam Physician 2012; 86: 1037-1041

[15] Ramshorn-Zimmer A et al. Weniger Non-COVID-19-Notfälle. Deutsches Ärzteblatt 2020; 117: A1201-A1205

[16] Bundesgesundheitsministerium: Abschlussbericht zur Überprüfung der Auswirkungen der Regelungen in den $\S 21$ bis 23 auf die wirtschaftliche Lage der Krankenhäuser durch den Beirat gemäß § 24 Krankenhausfinanzierungsgesetz, August 2020. https://www.bundesgesundheitsministerium.de/fileadmin/Dateien/3_Downloads/K/200821_Abschlussbericht_ des_Beirats_gemaess__24_KHG_Abschlussbericht_.pdf (zuletzt aufgesucht am 30.09.20)

[17] Hammoudeh AJ, Haft JI. Coronary-plaque rupture in acute coronary syndromes triggered by snow shoveling. N Engl J Med 1996; 335 : 2000-2001

[18] Strike PC, Perkins-Porras L, Whitehead DL et al. Triggering of acute coronary syndromes by physical exertion and anger: clinical and sociodemographic characteristics. Heart 2006; 92: 1035-1040 
[19] Follmer, Robert (2020): Mobilitätsreport 01, Ergebnisse aus Beobachtungen per repräsentativer Befragung und ergänzendem Mobilitätstracking bis Ende Mai, Ausgabe 29.05.2020, Bonn, Berlin. https:// www.bmbf.de/files/infas_Mobilit\%C3\%A4tsreport_20200610.pdf (zuletzt aufgesucht am 30.09.20)

[20] Huang H-H, Yen DH-T, Kao W-F et al. Declining Emergency Department Visits and Costs During the Severe Acute Respiratory Syndrome (SARS) Outbreak. Journal of the Formosan Medical Association 2006; 105: 31-37

[21] Pessoa-Amorim G, Camm CF, Gajendragadkar P et al. Admission of patients with STEMI since the outbreak of the COVID-19 pandemic: a survey by the European Society of Cardiology. Eur Heart J Qual Care Clin Outcomes 2020; 6: 210-216

[22] Wu J, Mamas M, Rashid M et al. Patient response, treatments and mortality for acute myocardial infarction during the COVID-19 pandemic. Eur Heart J Qual Care Clin Outcomes 2020; 1-9. https:// doi.org/10.1093/ehjqcco/qcaa062 (zuletzt aufgesucht am 30.09.20)

[23] Kirkup W, Merrick DW. A matter of life and death: population mortality and football results. J Epidemiol Community Health 2003; 57: 429-432

[24] Gonçalves-Pinho M, Mota P, Ribeiro J et al. The Impact of COVID-19 Pandemic on Psychiatric Emergency Department Visits - A Descriptive Study. Psychiatr Q. 2020; https://doi.org/10.1007/s11126-02009837-z (zuletzt aufgesucht am 30.09.20)

[25] Goldenberg MN, Parwani V. Psychiatric emergency department volume during Covid-19 pandemic. Am J Emerg Med. 2020; https:/| doi.org/10.1016/j.ajem.2020.05.088 (zuletzt aufgesucht am 30.09.20)

[26] Faltermaier T. Gesundheitspsychologie. Reihe: Grundriss der Psychologie, Band 21. Stuttgart: Kohlhammer; 2005: S. 193

[27] Waller H. Gesundheitswissenschaft: eine Einführung in Grundlagen und Praxis, 4. überarb. und erw.Aufl. Stuttgart: Kohlhammer; 2006: S. 14

[28] Helmert U. Subjektive Einschätzung der Gesundheit und Mortalitätsentwicklung. Gesundheitswesen 2003; 65: 47-54

[29] Lampert T, Müters S, Kuntz B et al. 30 Jahre nach dem Fall der Mauer: Regionale Unterschiede in der Gesundheit der Bevölkerung Deutschlands. Journal of Health Monitoring 2019; 4: 2-25

[30] Schmiedhofer M, Möckel M, Slagman A et al. Patient motives behind low-acuity visits to the emergency department in Germany: a qualitative study comparing urban and rural sites. BMJ Open 2016; 6: 1-9. https://bmjopen.bmj.com/content/bmjopen/6/11/e013323.full. pdf (zuletzt aufgesucht am 30.09.20)

[31] Bergmann-Klinikum: Experten haben Arbeit aufgenommen. Deutsches Ärzteblatt 2020. https://www.aerzteblatt.de/nachrichten/111941/ Bergmann-Klinikum-Experten-haben-Arbeit-aufgenommen (zuletzt aufgesucht am 30.09.20)

[32] Schlimpert V: Interview mit Prof. Dr. A. Zeiher: Wir müssen vorbereitet sein, damit wir Herzinfarkt-Patienten weiterhin behandeln können. 2020. https://www.kardiologie.org/covid-19/akutes-koronarsyndrom/wir-muessen-vorbereitet-sein--damit-wir-herzinfarkt-patienten-w/17896004 (zuletzt aufgesucht am 30.09.20)
[33] Landesamt für Gesundheit und Soziales Mecklenburg Vorpommern: Daten zur Corona-Pandemie: Lageberichte zur Coronavirus-Krankheit. (wöchentlich aktualisiert) 2020 https://www.lagus.mv-regierung.de/ Gesundheit/InfektionsschutzPraevention/Daten-Corona-Pandemie/ (zuletzt aufgesucht am 30.09.20)

[34] Deutscher Bundestag: Gesetz zum Schutz der Bevölkerung bei einer epidemischen Lage nationaler Tragweite. Bundesgesetzblatt 2020: 587-592

[35] Bundesregierung: Besprechung der Bundeskanzlerin mit den Regierungschefinnen und Regierungschefs der Länder am 12. März 2020. Berlin 2020. https://www.bundesregierung.de/breg-de/ themen/coronavirus/beschluss-zu-corona-1730292 (zuletzt aufgesucht am 30.09.20)

[36] Coronakrise: Gericht kippt Osterreiseverbot für Einwohner Mecklenburg-Vorpommerns. DER SPIEGEL 2020, 9. April 2020. https://www. spiegel.de/panorama/justiz/coronakrise-gericht-kippt-osterreiseverbot-fuer-einwohner-mecklenburg-vorpommerns-a-bc21f6bc59cf-4d8b-8317-dcb3f48dae43 (zuletzt aufgesucht am 30.09.20)

[37] Coronavirus in MV: Besuchsverbot im Kreiskrankenhaus Wolgast. Ostsee-Zeitung 2020, 15. März 2020. https://www.ostsee-zeitung.de/ Vorpommern/Usedom/Coronavirus-in-MV-Besuchsverbot-im-Kreiskrankenhaus-Wolgast (zuletzt aufgesucht am 30.09.20)

[38] Trotz Corona: Rostocker Mütter dürfen in Begleitung in den Kreißsaal. Ostsee-Zeitung 2020, 18. März 2020. https://www.ostsee-zeitung.de/ Mecklenburg/Rostock/Trotz-Corona-Rostocker-Muetter-duerfen-inBegleitung-in-den-Kreisssaal (zuletzt aufgesucht am 30.09.20)

[39] Coronavirus - Besucherinformationen. https://www.sana.de/ruegen/ gut-zu-wissen/coronavirus-besucherinformationen (zuletzt aufgesucht am 30.09.20)

[40] Onkologen warnen vor Bugwelle an zu spät diagnostizierten Krebsfällen. Deutsches Ärzteblatt 2020, 24 April 2020. https://www. aerzteblatt.de/nachrichten/112249 (zuletzt aufgesucht am 30.09.20)

[41] Nevermann NF, Hillebrandt KH, Knitter S et al. COVID-19 pandemic: implications on the surgical treatment of gastrointestinal and hepatopancreatobiliary tumours in Europe. Br J Surg 2020; 107: e 301-e302

[42] Falk Osterloh: Elf Prozent weniger Herzinfarkte 2020. Dtsch Arztebl 2020; 117: A-1658. B-1422

[43] Pressemitteilung Nr. 422 vom 27. Oktober 2020. Statistisches Bundesamt. https://www.destatis.de/DE/Presse/Pressemitteilungen/2020/10/PD20_422_46241.html (zuletzt aufgesucht am 20.12.2020)

[44] Giamello JD et al. The emergency department in the COVID-19 era. Who are we missing? Eur J Emerg Med 2020; 27: 305-306

[45] Baum A, Schwartz MD. Admissions to Veterans Affairs Hospitals for Emergency Conditions During the COVID-19 Pandemic. JAMA 2020; 324: $96-99$

[46] Holst J. COVID-19: Besondere Herausforderungen an Public Health in Theorie und Praxis. Gesundheitswesen 2020; 82: 829-835

[47] Heudorf U. COVID-19-Pandemie - Rationalität statt Regelungschaos tut not. Gesundheitswesen 2020; 82: 941-943 